\title{
The Application and Usage of Anesthetics in the Surgical Treatment of Orbital Cellulitis
}

\author{
Akshay Reddy ${ }^{1}$, Allen Dang ${ }^{2}$ and Himanshu Wagh ${ }^{2 *}$ \\ ${ }^{1}$ California Northstate University College of Health Sciences, Rancho Cordova, USA \\ ${ }^{2}$ California Northstate University College of Medicine, Elk Grove, USA
}

*Corresponding author: Himanshu Wagh, California Northstate University College of Medicine, Elk Grove, USA, Email: Himanshu.wagh5630@cnsu.edu

\section{ABSTRACT}

Keywords

Orbital cellulitis is a condition that can require surgery if severe symptoms progress Cellulitis to an undesirable state. Therefore, it is important for physicians to utilize anesthetics Orbital during these procedures to cause minimal harm to the patient. This review will analyze the consequences that the current application of anesthetics has on patients when being utilized in surgeries to eliminate orbital cellulitis. The authors find that Anesthetics when performing surgical intervention to terminate orbital cellulitis, physicians will Surgery favor bupivacaine, hyaluronidase, and lignocaine as anesthetics. Hyaluronidase is used to enhance the effects of the other two anesthetics, but can cause mild allergic reactions in certain patients. Additionally, this review seeks to analyze the current treatment of orbital cellulitis when surgical intervention is unnecessary. In mild cases of orbital cellulitis, the use of broad-range IV antibiotics in conjunction with oral antibiotics are sufficient remedies. The authors find that although surgical treatment of orbital cellulitis has improved over the years, there are still many changes that could be made to improve patient outcomes.

\section{INTRODUCTION}

Orbital cellulitis describes an infection in the soft tissues of the orbit. The pervasiveness of this disease can widely range between mild to severe and is treated accordingly with antimicrobials or surgery [1]. However, regardless of the severity of orbital cellulitis, immediate treatment is required as many complications can occur. Increased pressure in the orbit from orbital cellulitis can induce ocular complications and eventually lead to the development of meningitis and blindness if left untreated [1]. Mild orbital cellulitis typically presents clinical features of periorbital oedema, pain on eye movements, conjunctival chemosis, and reddening of the eyes. Key signs of orbital cellulitis include patient history of upper respiratory tract infection or sinusitis. Patients with orbital cellulitis undergo imaging and are given immediate intravenous broadspectrum antibiotics. Mild forms of orbital cellulitis are treated with initial intravenous antibiotics followed by four weeks of oral antibiotics. The commonest combination of intravenous antibiotics used are IV clindamycin + IV cephalosporin, IV cefuroxime, and IV cefotaxime + IV cloxacillin [2]. Severe forms of orbital cellulitis are indicated with progressive proptosis, globe displacement, and failure to respond to intravenous antibiotics. Additionally, orbital abscesses are crucial hallmarks for the need of more aggressive treatments [2,3]. Severe orbital cellulitis requires the use of surgical techniques to soothe complications intraorbital or intracranially [3]. Examples of techniques used include drainage of subperiosteal abscess or functional endoscopic sinus surgery (FESS). Both types of surgeries for orbital cellulitis are invasive in nature and induce excruciating pain to the patients. Thus the importance of anesthetics in these surgeries are crucial to the success of the operation and the quality of care given to the patient 


\section{Review Article}

\section{LITERATURE REVIEW}

Orbital cellulitis is the infection of the orbital tissues, specifically in the soft tissues posterior to the orbital septum. Globally, 1.6 out of 100,000 children and 0.1 per 100,000 adults are seen to develop orbital cellulitis [4]. Additionally, $1.3-5.6 \%$ of sinusitis incidences results in orbital cellulitis. Orbital cellulitis develops primarily from extensions of infection in the paranasal sinus, direct inoculation in the orbit following trauma, or hematogenous spread [5]. Examples of causes include surgical trauma, bacterial infections, and fungal infections . Despite having various routes of development, ethmoid sinusitis is the primary cause in almost $90 \%$ of all cases of orbital cellulitis [4]. The specific mechanism of progression involves edema of the sinus mucosa leading to the invasion of the edematous mucosa. Orbital cellulitis then develops when the organisms gain access to the orbit through the venous channels, foramina, or the orbital walls [1].

Although the prevalence figure may seem marginal, the complications that occur due to orbital cellulitis have serious consequences on the affected patient's quality of life. Prior to the development of modern antibiotics, contraction of orbital cellulitis resulted in mortality rates of up to $17 \%$ with $20 \%$ of survivors becoming blind in the affected eye [4]. Currently with modern medicine, orbital cellulitis results in blindness for $11 \%$ of cases and has lower rates of mortality [4]. However, additional intracranial complications stem from contraction of orbital cellulitis. Possible intracranial complications include cavernous sinus thrombosis, subperiosteal or orbital abscess formation, and meningitis [5]. Patients with complications of orbital cellulitis are promptly hospitalized and selected for either medical or surgical management. Typical medical management includes IV and oral antibiotic treatment [5]. In $12-15 \%$ of orbital cellulitis patients medical treatment alone will not suffice and will require forms of surgical management [4]. Generally, surgical treatment is performed on an emergency basis upon diagnosis of an orbital compartment syndrome, opacified sinuses, large subperiosteal abscess, or intraorbital abscess [5]. Further medical treatment will depend on subsequent follow-ups and information gathered through CT scans.

In order to perform the surgery for orbital cellulitis, a combination of anaesthetics are generally given to patients to reduce the pain that they may experience. The data reported in Table 1 suggests that physicians usually decide to inject the anesthetics into the patient's sub-Tenon space or their periorbital bulbar region [6-21]. This is most likely because these sites are usually closest to the site of infection. To prevent the patient from experiencing severe pain during surgery, anesthetics are typically applied near the site of the surgery. In previous years, general anesthesia, in the form of nitrous oxide,

Table 1: Classifications and Applications of Anesthetics Used During Cataract Surgery

\begin{tabular}{|c|c|c|c|}
\hline Author (year) & Type of anesthetic & Area (s) of anesthetic application & Classification of anesthetic (s) \\
\hline Bhat (2013) [6] & BV & Orbital rims & Local \\
\hline Dahlmann (2002) [7] & BN, LH, BV, HY & Sub-Tenon space & Local \\
\hline Kumar (2004) [8] & $\mathrm{OB}, \mathrm{LD}, \mathrm{LH}$ & $\begin{array}{c}\text { Topical, } \\
\text { Sub-Tenon space, } \\
\text { Peribulbar space }\end{array}$ & Local \\
\hline Liang (2006) [9] & HY, RH & Sub-Tenon space & Local \\
\hline Maheshwari (2009) [10] & PR, ET, KT & Preseptal space & General \\
\hline Morgan (2003) [11] & N/A & Sub-Tenon space & Local \\
\hline Mukherjee (2005) [12] & LH, BV & Peribulbar & Local \\
\hline Murray (1975) [13] & NO & Nasal cavity & General \\
\hline Park (2014) [14] & BV, LH, HY & Sub-Tenon space & Local \\
\hline Prasad (2011) [15] & PR, ET, KT & Sub-Tenon space & general \\
\hline Quhill (2004) [16] & BV, LH, HY & Peribulbar space & Local \\
\hline Raichura (2017) [17] & BV, LH, HY & Peribulbar space & Local \\
\hline Rajalakshmi (2016) [18] & $\mathrm{BV}, \mathrm{LH}, \mathrm{HY}$ & Peribulbar space & Local \\
\hline Redmill (2001) [19] & N/A & Sub-Tenon space & Local \\
\hline Saha (2017) [20] & $\mathrm{N} / \mathrm{A}$ & Intravitreal space & General \\
\hline Varma (2003) [21] & $\mathrm{BV}, \mathrm{LH}, \mathrm{HY}$ & Posterior chamber & Local \\
\hline
\end{tabular}

BN: benoxinate, BV: bupivacaine, HY: hyaluronidase, LD: lidocaine, LH: lignocaine hydrochloride, OB: Oxybuprocaine, RH: ropivacaine hydrochloride, PR: Propofol, ET: etomidate, KT: ketamine, NO: nitrous oxide 
was given to patients when this surgery was being conducted [13]. However, this potentially could have been dangerous as exposure to nitrous oxide can lead to negative side effects such as: loss of balance, impaired memory, and even dissociation [22]. Thankfully, medical science has improved over the years and nitrous oxide is no longer used as an anesthetic for this procedure. Based on the data collected from the literature, that was reported in Table 1 , most physicians usually administer a combination of bupivacaine, hyaluronidase, and lignocaine to patients before they undergo the necessary surgical procedures to treat their severe cases of orbital cellulitis [6-21]. Application of these pharmaceuticals functions as a local anesthetic for the orbital during surgery [6]. This is beneficial to the patient as general anesthesia can affect multiple bodily systems and cause negative side effects such as: nausea, urinary retention, and dizziness $[23,24]$. In fact within the literature, we could only find three instances where general anesthesia was used instead of local anesthesia for surgical procedures involving orbital cellulitis $[10,13,15]$.

Although a majority of procedures for treating orbital cellulitis involve the combination of the aforementioned drugs, there are instances when hyaluronidase is substituted for a different pharmaceutical, or when it is simply not used in combination with the other two anesthetics $[6,8,12]$. This could potentially be due to the fact that there have been instances where patients proved to be allergic to hyaluronidase. The side effects that allergic patients experience when exposed to hyaluronidase are mild cases of temporary swelling and itching [25]. Numerous physicians prefer to use hyaluronidase when possible as the enzyme helps spread the other anesthetics to ensure that their effects on the area are maximized. Although hyaluronidase is still currently used in many procedures, it may one day be replaced by oxybuprocaine. In one of the studies within the literature, it was shown that the analgesic effects of the combination of bupivacaine, lignocaine, and oxybuprocaine were able to achieve similar patient outcomes [8]. Perhaps in the future, hyaluronidase may be phased out, but further research needs to be conducted in order to determine both the short and long term effects of oxybuprocaine.

Despite all of the progress that has been made in order to understand how to effectively use medications and interventional surgery to treat severe orbital cellulitis. Additional research is needed in order to determine more ideal medications that can be used for these procedures in order to minimize side effects that patients may experience. Currently we understand that hyaluronidase can cause an allergic reaction with certain patients, but we do not have a clear understanding about why this occurs. Supplementary research involving chemical analysis and Western blot techniques may be required in order to answer this question [26,27]. Agents that help stabilize the integrity of red blood cells and anti-body proteins, such as EDTA, may be needed when conducting this research to give a clear understanding for why hyaluronidase can cause irritation in certain patients [28-30]. Although treatment options exist for severe cases of orbital cellulitis, it would be more ideal if more cases of orbital cellulitis were caught before they progressed to severe stages. Part of the reason that so many cases of orbital cellulitis reach a severe state of progression is because many patients who have the condition go undiagnosed for long periods of time [31]. Perhaps, in the future, artificial intelligence software could be developed to help increase the rate at which patients can get diagnosed for diseases such as orbital cellulitis [32-33]. In order to reach this point, further research utilizing imaging technology and data collection amongst the patient population may be necessary.

\section{CONCLUSION}

Orbital cellulitis is an infectious disease that can present mild to severe complications. When the complications become severe, surgical treatment may be required to handle the disease. It is important to select the right anesthetics to use when performing the procedure to reduce patient harm. Physicians will usually apply the anesthetic on the peribulbar or sub-Tenon region of the patient before they begin the surgical procedure. Currently the most popular anesthetics used for this procedure are bupivacaine, hyaluronidase, and lignocaine which can be applied locally. However, hyaluronidase can trigger allergic reactions within some patients. Therefore further research into other anesthetics such as oxybuprocaine, is needed in order to improve the current standard of care for patients who undergo surgical procedures for orbital cellulitis. Future studies should also be organized to determine why certain individuals experience an allergic reaction when they are exposed to hyaluronidase.

\section{REFERENCES}

1. Babar TF, Zaman M, Khan MN, Khan MD: Risk factors of preseptal and orbital cellulitis. J Coll Physicians Surg Pak. 2009, 19:39-42.

2. Garcia GH, Harris GJ. Criteria for nonsurgical management of subperiosteal abscess of the orbit: analysis of outcomes 1988-1998. Ophthalmology. 2000, 8:1454-6. 10.1016/s0161642000242-6. PMID: 10919887

3. Chiu ES, Capell BC, Press R, Aston SJ, Jelks EB, Jelks GW. Successful management of orbital cellulitis and temporary visual loss after blepharoplasty. Plast Reconstr Surg. 2006, 118:95. 10.1097/01.prs.0000233031.17109.c6. Erratum in: Plast Reconstr

4. Mowatt L. Orbital cellulitis. Challenging Issues on Paranasal Sinuses. Intechopen, 10.5772/intechopen.79983. 2018. 10.5772/ intechopen.79983 


\section{Review Article}

5. McKinley SH, Yen MT, Miller AM, Yen KG: Microbiology of pediatric orbital cellulitis. Am J Ophthalmol. 2007, 144:497-501. 10.1016/j.ajo.2007.04.049

6. Bhat MT, Hegde HV, Santhosh MC, Rao RP. Orbital exenteration under trigeminal block: An innovative method of regional anesthesia Saudi J Anaesth. 2013, 7:470-3. 10.4103/1658-354X.121051

7. Dahlmann AH, Appaswamy S, Headon MP. Orbital cellulitis following sub-Tenon's anaesthesia. Eye (Lond. 2002, 16:200-1.

8. Kumar CM, Dowd TC, Dodds C, Boyce R. Orbital swelling following peribulbar and sub-Tenon's anaesthesia. Eye (Lond. 2004, 18:418-20. 10.1038/sj.eye.6700673

9. Liang SY, Moloney G, O’Donnell BA, Fernando G. Orbital cellulitis as a postoperative complication of sub-Tenon anaesthesia in cataract surgery. Clin Exp Ophthalmol. 2006, 34:897-9. 10.1111/j.1442-9071.2006.01361.x

10. Maheshwari R, Maheshwari S, Shah T. Acute dacryocystitis causing orbital cellulitis and abscess. 28:196-9. 10.1080/01676830902925529. PMID: 19839913

11. Morgan SJ. Orbital cellulitis following corneal gluing under subTenon's local anaesthesia. Eye (Lond. 2003, 17:284-5. 10.1038/ sj.eye. 6700279

12. Mukherjee C, Mitra A, Mushtaq B. Orbital cellulits following cataract surgery under peribulbar anaesthesia. GMS Ophthalmol Cases. 2015165, 2:10. 10.3205/oc000024

13. Murray JE, Swanson LT, Strand RD, Hricko GM. Evaluation of craniofacial surgery in the treatment of facial deformities. Ann Surg. 1975, 182:240-65. 10.1097/00000658-197509000-00007. PMID: 169753 PMCID: PMC1343932

14. Park S, Lim LT. Orbital inflammation secondary to a delayed hypersensitivity reaction to sub-Tenon's hyaluronidase. Semin Ophthalmol. 2014, 29:57-8. 10.3109/08820538.2012.760616. Epub 2013 Jul 19. PMID: 23869964

15. Srinivasa Prasad B, Govardhan T. A rare case of orbital cellulitis followed by therapeutic(orthodontic) extraction. J Maxillofac Oral Surg. 2011, 10:257-61. 10.1007/s12663-010-0150-4. Epub 2011 Mar 22. PMID: 22942598 PMCID: PMC3238550

16. Quhill F, Bowling B, Packard RB. Hyaluronidase allergy after peribulbar anesthesia with orbital inflammation. J Cataract Refract Surg. 2004, 30:916-7. 10.1016/j.jcrs.2003.08.024. PMID: 15093662

17. Raichura ND, Alam MS, Jaichandran VV, Mistry S, Mukherjee B: Hyaluronidase allergy mimicking orbital cellulitis. Orbit. 2018, 37:149-153. 10.1080/01676830.2017.1383465. Epub 2017 Oct 20. PMID: 29053033

18. Rajalakshmi AR, Kumar MA. Hyaluronidase hypersensitivity: A rare complication of peribulbar block. Indian J Ophthalmol. 2016, 64:160-2. 10.4103/0301-4738.179717

19. Redmill B, Sandy C, Rose GE. Orbital cellulitis following corneal gluing under sub-Tenon's local anaesthesia. Eye (Lond. 2001, 15:554-6. 10.1038/eye.2001.178. PMID: 11767042

20. Saha BC, Kumari R, Sinha BP. Clinical Outcome of Probing in Infants with Acute Dacryocystitis - A Prospective Study. J Clin Diagn Res. 2017, 11:01-03. 10.7860/JCDR/2017/29033.10295. Epub 2017 Aug 1. PMID: 28969168 PMCID: PMC5620809
21. Varma D, Metcalfe TW. Orbital cellulitis after peribulbar anaesthesia for cataract surgery. Eye (Lond. 2003, 17:105-6. $10.1038 /$ sj.eye. 6700238

22. van Amsterdam J, Nabben T, van den Brink W. Recreational nitrous oxide use: Prevalence and risks. Regul Toxicol Pharmacol. 2015, 73:790-6. 10.1016/j.yrtph.2015.10.017. Epub 2015 Oct 22. PMID: 26496821

23. Smith G, D'Cruz JR, Rondeau B, et al. General Anesthesia for Surgeons. [Updated 2021 Aug 12]. In. StatPearls [Internet, Treasure Island (FL): StatPearls Publishing; 2021.

24. Reddy AJ, Tak N, Martel JB. Case study of endogenous streptococcal endophthalmitis in the critical care setting. Cureus. 2021, 5:16192. 10.7759/cureus. 16192

25. Kim TW, Lee JH, Yoon KB, Yoon DM. Allergic reactions to hyaluronidase in pain management -A report of three cases-. Korean J Anesthesiol. 2011, 60:57-9. 10.4097/kjae.2011.60.1.57. Epub 2011 Jan 28. PMID: 21359084 PMCID: PMC3040435

26. Tak N, Wagh P, Sandhu S, Reddy A, Wagh H. A quantitative analysis on the effect of varying nitrate concentrations on $\mathrm{pH}$ levels on the growth of algae. Appl Cell Biol. 2021, 9:24-28. 10.53043/2320-1991.acb90005

27. Sandhu S, Sandhu A, Reddy AJ, et al. An investigative study into the C. fluminea and its interactions with its surrounding ecosystem. Appl Cell Biol. 2021, 1:33-42. 10.53043/2320-1991. acb90007

28. Sunil A, Shaheed G, Reddy AJ, Nawathey N, Brahmbhatt H. A review on the role of ethylenediaminetetraacetic acid (EDTA) in the treatment and understanding of psoriasis. Cureus. 2021, $16: 16424$. 10.7759 /cureus. 16424

29. Senthilraja V, Lou E, Nakka A, et al. Exploring Drug and AntibodyBased Treatment Options for Creutzfeldt-Jakob Disease. Appl Cell Biol. 2021:43-48. 10.53043/2320-1991.acb90008

30. Wagh H, Reddy AJ. A comparison of DASH scores resulting from different treatment options for the intra-articular distal radius fracture in the geriatric population. Appl Cell Biol. 2021, 9:18-23. 10.53043/2320-1991.acb90004

31. Danishyar A, Sergent SR. Orbital Cellulitis. [Updated 2021 Aug 12]. In. StatPearls [Internet, Treasure Island (FL): StatPearls Publishing; 2021.

32. Reddy AJ, Martel JB. Deep neural network learning for detection and grading of diabetic retinopathy. Appl Cell. 2020, 8:67-73. 10.53043/2320-1991.acb90001

33. Tak N, Reddy A J, Martel J, et al. (August 30, 2021) Clinical WideField Retinal Image Deep Learning Classification of Exudative and Non-Exudative Age-Related Macular Degeneration. Cureus. 13:17579. 10.7759/cureus. 17579

Citation: Reddy A, Dang A, Wagh H (2021) The Application and Usage of Anesthetics in the Surgical Treatment of Orbital Cellulitis. Appl Cell Biol, 9(3), 2021 [81-84] 